\title{
Evaluation of the relationship between disease activity and serum bilirubin, albumin, and uric acid levels in Crohn's disease
}

\author{
(1)Gizem Bedir Keser ${ }^{1}$, @Nergiz Ekmen ${ }^{2}$ \\ ${ }^{1}$ Gazi University, Department of Internal Medicine, Faculty of Medicine, Ankara, Turkey \\ ${ }^{2}$ Gazi University, Faculty of Medicine, Department of Internal Medicine, Division of Gastroenterology, Ankara, Turkey
}

Cite this article as: Bedir Keser G, Ekmen N. Evaluation of the relationship between disease activity and serum bilirubin, albumin, and uric acid levels in Crohn's disease. J Health Sci Med 2021; 4(5): 639-645.

\begin{abstract}
Objective: Oxidative stress and antioxidant deficiency play key roles in the pathogenesis of gastrointestinal damage associated with Crohn's disease (CD). Serum bilirubin, uric acid (UA) and albumin are non-enzymatic antioxidants that play a role in oxidative stress control. In this study, it was aimed to evaluate the change in serum bilirubin, UA and albumin levels due to disease activity.

Material and Method: This study was designed as a single-center, cross-sectional and retrospective. The clinical and demographic data of the patients, disease activity, as well as serum albumin, UA, and bilirubin values were recorded from the hospital database. Study variables were statistically analyzed between patient and control groups based on disease activity.

Results: A total of 234 people, 114 with CD and 120 with controls, were included in the study. Total bilirubin, direct bilirubin and albumin levels were found to be significantly lower in the CD than in the health control $(p=0.045, p<0.001, p<0.001)$. There was a significant difference in total bilirubin, direct bilirubin, indirect bilirubin and albumin levels between the control group and active CD ( $\mathrm{p}=0.009 ; \mathrm{p}=0.001 ; \mathrm{p}=0.037 ; \mathrm{p}<0.001)$; no significant difference with the UA level ( $\mathrm{p}=0.992)$. There was a significant difference in direct bilirubin levels between the control group and remission $\mathrm{CD}$ ( $\mathrm{p}=0.005)$; no difference in total bilirubin, indirect bilirubin, albumin, and UA levels ( $\mathrm{p}=0.263 ; \mathrm{p}=0.440 ; \mathrm{p}=0.112 ; \mathrm{p}=0.365$, respectively).

Conclusion: Antioxidant capacity reaches levels similar to the healthy population in patients who achieve remission with medical treatment.
\end{abstract}

Keywords: Albumin, bilirubin, Crohn's disease, oxidative stress, uric acid

\section{INTRODUCTION}

Crohn's disease $(\mathrm{CD})$ is a chronic inflammatory disease that can affect the gastrointestinal tract from mouth to rectum (1). Its annual incidence varies according to geographical region, environmental factors, genetic predisposition and lifestyle (2). With the increasing incidence of inflammatory bowel disease, the diagnosis and treatment of $\mathrm{CD}$ has gained importance in terms of gastroenterology (3). Since the etiology of the disease has not been fully elucidated, the goal of treatment is to convert the disease from active to remission. Multiple laboratory indices are used to diagnose and evaluate the activity of $\mathrm{CD}$. Some laboratory parameters such as C-reactive protein (CRP), erythrocyte sedimentation rate (ESR), red cell distribution width (RDW), tumor necrosis factor, and fecal calprotectin were found to be associated with the activity of the disease (4).
Such laboratory tests not only show the activity of the disease, but may also be indicative of existing bacterial infection. Therefore, there is a need for low-cost and noninvasive tests that can evaluate the activity of the disease, with high patient compliance.

Bilirubin, the end product of heme metabolism, is a potent endogenous antioxidant (5). While bilirubin was previously considered as a cytotoxic waste with potential toxicity, it has recently come to the fore with its strong antioxidant, anti-inflammatory and immunosuppressive properties (5). The antioxidant property of bilirubin has been demonstrated in patients with systemic lupus erythematosus (6) and polymyositis (7). Uric acid (UA) is the end product of purine catabolism and has been shown to scavenge more than half of the free radicals in 
the blood as an antioxidant (8). The antioxidant role of UA was demonstrated in a study conducted with patients with myasthenia gravis (9). Serum albumin is known as an antioxidant against oxidative damage.

Considering the role of serum bilirubin, UA and albumin in inflammatory diseases, we aimed to investigate the relationship between these values and the activity of CD.

\section{MATERIAL AND METHOD}

The study was approved by Gazi University Faculty of Medicine, Clinical Research Ethics Committee (Date: 7/12/2020, Decision No: 834). All procedures were carried out in accordance with the ethical rules and the principles of the Declaration of Helsinki.

\section{Patient Selection}

This study is a single-center retrospective cohort study, and patients with $\mathrm{CD}$ who applied to the gastroenterology outpatient clinic between 1 October 2015 and 1 October 2020 were included. When the sample was calculated with $95 \%$ confidence interval and $80 \%$ power of analysis, the sample size was calculated as 87 people. Our study included patients aged 18 years and older, who were diagnosed with Crohn's disease based on medical history, clinical features, endoscopic, histopathological, imaging methods and laboratory tests, and whose follow-up and medical treatment continued. The healthy control group was selected from the patients who applied to the general internal medicine outpatient clinic in the same period.

\section{Determination of Study Variables and Disease Activity}

In the anamnesis form obtained from the hospital database of the patients diagnosed with Crohn's disease; gender, age, date of diagnosis, localization of the disease, presence of systemic disease, extraintestinal findings, documentation of current and past medical treatment were recorded. Serum total bilirubin, direct bilirubin, indirect bilirubin, gamma glutamyltransferase (GGT), alkaline phosphatase (ALP), alanine aminotransferase (ALT), aspartate aminotransferase (AST), albumin, UA, CRP, ESR, RDW, globulin, hemoglobin, hematocrit, white blood cell, lymphocyte, neutrophil, monocytes, ferritin, mean platelet volume (MPV) values were examined. Disease activity was determined at the time of admission to the hospital using the information in the anamnesis form obtained from the database, clinical features and laboratory parameters, and the CD activity index (CDAI) score. Patients with a CDAI score below 150 were considered in remission, 150-220: mild-moderate, 221450: moderate-severe, $>450$ : severe fulminant Crohn's disease. Age at diagnosis, site of involvement, type of disease and presence of perianal disease were determined according to the Montreal classification.

\section{Statistical Analysis}

Statistical analyzes were performed using the SPSS for Windows Version 22. Frequencies were stated for the variables in the categorized data (qualitative), and for the numerical data (quantitative) variables, the means \pm standard deviation if appropriate for the normal distribution, and the median (min-max) values if not for the normal distribution. Whether the variables fit the normal distribution or not was evaluated with the Kolmogorov Smirnov test. Parametric tests (Independent Sample $t$ Test) were used for the variables showing normal distribution, and nonparametric tests (Chi-Square, Mann Withney U Test) were used for those that did not. Kruskal Wallis Test was used for comparison of triple numeric data. Spearman correlation test was used for the correlation between continuous variables. The statistical significance of this study was accepted as $\mathrm{p} \leq 0.05$.

\section{RESULTS}

A total of 234 people, 114 with $\mathrm{CD}$ and 120 with controls, were included in the study. $49 \%(n=56)$ of CD and $39 \%$ $(n=47)$ of the healthy control group were male. There was no statistically significant difference between the two groups by gender ( $\mathrm{p}=0.125$ ). There was no statistically significant difference in the mean age of the $\mathrm{CD}(39.36 \pm$ $12.26)$ and control group $(39.12 \pm 13.63)(\mathrm{p}=0.886)$. While $28.9 \%(n=33)$ of $C D$ were in the active period, $71.1 \%$ $(\mathrm{n}=81)$ were in remission, and the clinical demographic characteristics of the entire group are summarized in Table 1.

There was a statistically significant difference in total bilirubin, direct bilirubin, albumin, globulin, ALP, ferritin, hemoglobin, neutrophil, lymphocyte, platelet, RDW and MPV values between the CD and control group $(\mathrm{p}<0.05)$ (Table 2$)$. There was no statistically significant difference between indirect bilirubin, UA, AST, ALT, GGT, leukocytes, monocytes, and hematocrit values $(\mathrm{p}>0.05)$ (Table 2).

A statistically significant difference was found between CRP and albumin levels between remission and active CD $(p<0.001, p<0.001)$. No statistically significant difference was found between gender, age, total bilirubin, direct bilirubin, indirect bilirubin and UA levels $(\mathrm{p}=0.361, \mathrm{p}=0.476, \mathrm{p}=0.170, \mathrm{p}=0.280, \mathrm{p}=0.233, \mathrm{p}=0.478$, respectively) (Table 3 ) .

There was a statistically significant difference between the control group and active CD, levels of total bilirubin, direct bilirubin, indirect bilirubin, and albumin $(\mathrm{p}=0.009, \mathrm{p}=0.001, \mathrm{p}=0.037, \mathrm{p}<0.001$, respectively). There was no statistically significant difference between the UA level ( $\mathrm{p}=0.992)$ (Table 4). There was a statistically significant difference between direct bilirubin levels 
between control and remission CD $(\mathrm{p}=0.005)$. No statistically significant difference was found between total bilirubin, indirect bilirubin, albumin, and UA levels ( $>0.05)$ (Table 4).

There was a statistically significant difference in terms of disease activity severity (mild, moderate and severe), albumin and CRP $(\mathrm{p}<0.001, \mathrm{p}<0.001$, respectively). There was no statistically significant difference between total bilirubin, direct bilirubin, indirect bilirubin and UA levels $(\mathrm{p}=0.300, \mathrm{p}=0.378, \mathrm{p}=0.340, \mathrm{p}=0.173$, respectively).

\begin{tabular}{|c|c|c|}
\hline \multirow[b]{2}{*}{ Disease activity } & \multicolumn{2}{|c|}{$\mathrm{CD}, \mathrm{n}=114$} \\
\hline & $\mathbf{n}$ & $\%$ \\
\hline Active & 33 & 28.9 \\
\hline Remission & 81 & 71.1 \\
\hline \multicolumn{3}{|l|}{ Gender } \\
\hline Male & 56 & 49 \\
\hline Female & 58 & 51 \\
\hline \multicolumn{3}{|l|}{ Comorbidities } \\
\hline Yes & 30 & 26.4 \\
\hline No & 84 & 73.6 \\
\hline \multicolumn{3}{|l|}{ Age at diagnosis } \\
\hline$<16$ years $(\mathrm{A} 1)$ & 7 & 6.1 \\
\hline $17-40$ years (A2) & 78 & 68.4 \\
\hline$>40$ years $(\mathrm{A} 3)$ & 29 & 25.5 \\
\hline \multicolumn{3}{|l|}{ Disease localization } \\
\hline Ileal (L1) & 32 & 29.6 \\
\hline Colonic (L2) & 36 & 33.3 \\
\hline Ileocolonic (L3) & 38 & 35.2 \\
\hline Upper GIS involvement (L4) & 2 & 1.9 \\
\hline \multicolumn{3}{|l|}{ Disease Behaviour } \\
\hline Inflammatory (B1) & 62 & 54.85 \\
\hline Obstructive/stenosing (B2) & 19 & 16.85 \\
\hline Penetrating/fistulizing (B3) & 20 & 17.65 \\
\hline Perianal (P) & 12 & 10.65 \\
\hline \multicolumn{3}{|l|}{ Extraintestinal symptom } \\
\hline Yes & 24 & 20.5 \\
\hline No & 90 & 79.5 \\
\hline \multicolumn{3}{|l|}{ Disease activity (CDAI score) } \\
\hline$<150$ remission & 81 & 71.1 \\
\hline $150-220$ mild moderate & 23 & 20.2 \\
\hline 220-450 moderate-severe & 10 & 8.8 \\
\hline$>450$ fulminant & 0 & 0 \\
\hline \multicolumn{3}{|l|}{ Type of treatment } \\
\hline Vedolizumab/Certolizumab & 13 & 11.4 \\
\hline 5-ASA & 19 & 16.6 \\
\hline Azathioprine \pm anti TNF & 37 & 32.4 \\
\hline Prednol or unknown & 8 & 7.0 \\
\hline Azathioprine \pm 5 -ASA & 37 & 32.4 \\
\hline Only azathioprine treatment & 18 & 15.7 \\
\hline Other treatments & 96 & 84.2 \\
\hline $\mathrm{CD}=$ Crohn's disease & & \\
\hline
\end{tabular}

When the laboratory parameters of CD patients who received only azathioprine treatment and those who received other treatment types were compared, no statistically significant difference was found between total bilirubin, direct bilirubin, indirect bilirubin, albumin, and UA values ( $\mathrm{p}>0.05)$ (Table 5).

No correlation was found between the duration of treatment and serum total bilirubin, direct bilirubin, indirect bilirubin, UA, and albumin values $(\mathrm{p}=0.845$, $\mathrm{r}=0.019 ; \mathrm{p}=0.728, \mathrm{r}=0.033 ; \mathrm{p}=0.997 \mathrm{r}=0.000 ; \mathrm{p}=0.894$, $\mathrm{r}=0.013 ; \mathrm{p}=0.965 \mathrm{r}=0.004$, respectively).

\section{Table 2. Comparison of demographic characteristics and} laboratory parameters in the study group

\begin{tabular}{|c|c|c|c|}
\hline & $C D, n=114$ & Control, $n=120$ & $\mathbf{P}$ \\
\hline Gender n (\%) & & & 0.125 \\
\hline Male & $56(49 \%)$ & 47 (39\%) & \\
\hline Female & $58(51 \%)$ & $73(61 \%)$ & \\
\hline Age (years)* & $39.36 \pm 12.26$ & $39.12 \pm 13.63$ & 0.886 \\
\hline $\mathrm{CRP}, \mathrm{mg} / \mathrm{L}^{* *}$ & $7.30(1-206)$ & & \\
\hline $\begin{array}{l}\text { Total bilirubin, } \\
\mathrm{mg} / \mathrm{dL}\end{array}$ & $\begin{array}{c}0.52 \\
(0.13-2.45)\end{array}$ & $\begin{array}{c}0.58 \\
(0.26-2.37)\end{array}$ & 0.045 \\
\hline $\begin{array}{l}\text { Direct bilirubin, } \\
\mathrm{mg} / \mathrm{dL}\end{array}$ & $\begin{array}{c}0.10 \\
(0.03-0.37)\end{array}$ & $\begin{array}{c}0.13 \\
(0.05-0.51)\end{array}$ & $<0.001$ \\
\hline $\begin{array}{l}\text { Indirect bilirubin, } \\
\mathrm{mg} / \mathrm{dL}\end{array}$ & $\begin{array}{c}0.42 \\
(0.05-2.14)\end{array}$ & $\begin{array}{c}0.46 \\
(0.11-1.86)\end{array}$ & 0.128 \\
\hline Albumin, g/dL & $4.30(1.50-8.40)$ & $4.50(3.60-5.10)$ & $<0.001$ \\
\hline $\mathrm{UA}, \mathrm{mg} / \mathrm{dL}$ & $4.85(2.20-8.80)$ & $4.70(2.20-8.30)$ & 0.481 \\
\hline AST, U/L & $19.00(10-225)$ & $20.00(11-42)$ & 0.477 \\
\hline ALT, U/L & $16.00(2-271)$ & $17.00(7-69)$ & 0.486 \\
\hline ALP, U/L & $90.00(47-230)$ & $77.50(38-164)$ & 0.003 \\
\hline GGT, U/L & $22.00(7-277)$ & $18.00(8-127)$ & 0.054 \\
\hline Globulin, g/dL & $3.15(2.30-4.70)$ & $2.90(2.30-3.90)$ & $<0.001$ \\
\hline Ferritin, ng/mL & $35.00(2-595)$ & $21.00(2-175)$ & 0.007 \\
\hline $\begin{array}{l}\text { Hemoglobin, } \\
\text { g/dL }\end{array}$ & $\begin{array}{c}13.60 \\
(7.40-18.10)\end{array}$ & $\begin{array}{c}13.95 \\
(10.00-18.30)\end{array}$ & 0.007 \\
\hline $\begin{array}{l}\text { Leukocyte, } \\
\times 10^{3} / \mathrm{uL}\end{array}$ & $\begin{array}{c}7380.00 \\
(3110-22620)\end{array}$ & $\begin{array}{c}7100.00 \\
(3790-12720)\end{array}$ & 0.140 \\
\hline $\begin{array}{l}\text { Neutrophil, } \\
\times 10^{3} / \mathrm{uL}\end{array}$ & $\begin{array}{c}4425.00 \\
(1150-18450)\end{array}$ & $\begin{array}{c}3960.00 \\
(670-7170)\end{array}$ & 0.002 \\
\hline $\begin{array}{l}\text { Lymphocyte, } \\
\times 10^{3} / \mathrm{uL}\end{array}$ & $\begin{array}{c}2035.00 \\
(300-4550)\end{array}$ & $\begin{array}{c}2275.00 \\
(990-4770)\end{array}$ & 0.002 \\
\hline $\begin{array}{l}\text { Monocyte, } \\
\times 10^{3} / \mathrm{uL}\end{array}$ & $\begin{array}{c}560.00 \\
(120-1610)\end{array}$ & $\begin{array}{c}560.00 \\
(230-1140)\end{array}$ & 0.488 \\
\hline $\begin{array}{l}\text { Platelet, } \\
\times 10^{3} / \mathrm{uL}\end{array}$ & $\begin{array}{c}303000.00 \\
(110000-766000)\end{array}$ & $\begin{array}{c}276500.00 \\
(163000-439000)\end{array}$ & 0.001 \\
\hline RDW, \% & $13.65(12-32)$ & $13.00(11-19)$ & $<0.001$ \\
\hline MPV, fL & $9.70(8.10-21.70)$ & $\begin{array}{l}10.20(8.40- \\
12.90)\end{array}$ & 0.002 \\
\hline Hematocrit, \% & $41.50(22-53)$ & $42.05(34-54)$ & 0.174 \\
\hline $\begin{array}{l}\text { Sedimentation, } \\
\mathrm{mm} / \mathrm{h}\end{array}$ & $\begin{array}{l}20.50 \\
(2-105)\end{array}$ & & \\
\hline \multicolumn{4}{|c|}{$\begin{array}{l}{ }^{*} \text { mean } \pm \text { standard deviation. }{ }^{* *} \text { median }(\min -\mathrm{max}) . \mathrm{CD}=\mathrm{Crohn}{ }^{\prime} \text { disease, } \mathrm{ALT}=\text { alanine } \\
\text { aminotransferase. ALP=alkaline phosphatase. AST=aspartate aminotransferase. } \\
\mathrm{CRP}=\mathrm{C} \text { reactive protein. } \mathrm{GGT}=\text { gammaglutamyl transferase. MPV=mean platelet } \\
\text { volume. RDW= red cell distribution width. } \mathrm{UA}=\text { uric acid }\end{array}$} \\
\hline
\end{tabular}




\begin{tabular}{|c|c|c|c|}
\hline & Remission CD, $\mathrm{n}=81$ & Active CD, n=33 & $\mathbf{P}$ \\
\hline \multicolumn{4}{|l|}{ Gender n (\%) } \\
\hline Male & $42(51.9)$ & $14(42.4)$ & 0.361 \\
\hline Female & $39(48.1)$ & $19(57.6)$ & \\
\hline Age (years)* & $39.77 \pm 12.03$ & $38.36 \pm 12.95$ & 0.476 \\
\hline CRP, $\mathrm{mg} / \mathrm{L}^{* *}$ & $47.37(1-73)$ & $78.36(12-105)$ & $<0.001$ \\
\hline Total bilirubin, mg/dL & $0.52(0.13-2.45)$ & $0.50(0.26-0.83)$ & 0.170 \\
\hline Direct bilirubin, mg/dL & $0.11(0.03-0.37)$ & $0.09(0.06-0.19)$ & 0.280 \\
\hline Indirect bilirubin, $\mathrm{mg} / \mathrm{dL}$ & $0.42(0.05-2.14)$ & $0.40(0.20-0.66)$ & 0.233 \\
\hline Albumin, g/dL & $4.40(1.50-8.40)$ & $4.00(2.90-7.80)$ & $<0.001$ \\
\hline $\mathrm{UA}, \mathrm{mg} / \mathrm{dL}$ & $4.95(2.30-8.80)$ & $4.45(2.20-7.70)$ & 0.478 \\
\hline
\end{tabular}

\begin{tabular}{|c|c|c|c|c|c|}
\hline & Control, $n=120$ & Active CD, $n=33$ & $\mathbf{P}$ & Remission CD, $n=81$ & $\mathbf{P}$ \\
\hline Total bilirubin,mg/dL* & $0.58(0.26-2.37)$ & $0.50(0.26-0.83)$ & 0.009 & $0.52(0.13-2.45)$ & 0.263 \\
\hline Direct bilirubin,mg/dL & $0.13(0.05-0.51)$ & $0.09(0.06-0.19)$ & 0.001 & $0.11(0.03-0.37)$ & 0.005 \\
\hline Indirect bilirubin, $\mathrm{mg} / \mathrm{dL}$ & $0.46(0.11-1.86)$ & $0.40(0.20-0.66)$ & 0.037 & $0.42(0.05-2.14)$ & 0.440 \\
\hline Albumin, g/dL & $4.50(3.60-5.10)$ & $4.00(2.90-7.80)$ & $<0.001$ & $4.40(1.50-8.40)$ & 0.112 \\
\hline UA. $\mathrm{mg} / \mathrm{dL}$ & $4.70(2.20-8.30)$ & $4.45(2.20-7.70)$ & 0.992 & $4.95(2.30-8.80)$ & 0.365 \\
\hline
\end{tabular}

\begin{tabular}{|c|c|c|c|}
\hline & Only azathioprine treatment, $n=18$ & Other treatments, $n=96$ & $\mathbf{P}$ \\
\hline Total bilirubin $\mathrm{mg} / \mathrm{dL}^{*}$ & $0.52(0.23-1.35)$ & $0.52(0.13-2.45)$ & 0.887 \\
\hline Direct bilirubin $\mathrm{mg} / \mathrm{dL}$ & $0.10(0.05-0.26)$ & $0.11(0.03-0.37)$ & 0.832 \\
\hline Indirect bilirubin $\mathrm{mg} / \mathrm{dL}$ & $0.40(0.09-0.66)$ & $0.42(0.05-2.14)$ & 0.657 \\
\hline Albumin g/dL & $4.30(3.90-4.80)$ & $4.30(1.50-8.40)$ & 0.617 \\
\hline UA. $\mathrm{mg} / \mathrm{dL}$ & $4.50(2.60-6.90)$ & $4.90(2.20-8.80)$ & 0.317 \\
\hline
\end{tabular}

\section{DISCUSSION}

To the best of our knowleged, our study is the first reported from Turkey to examine the relationship between serum bilirubin, albumin and UA which are non-enzimatic antioxidant molecules and disease activity in patients with CD followed up with medical treatment.

In the current study, found that serum total bilirubin and albumin levels, which reflect the change in serum antioxidant capacity due to the increase in inflammationrelated oxidative stress, were significantly lower in CD compared to the healthy controls. But there was no significant difference in indirect bilirubin and UA levels.

In the study of Su et al. (10) serum bilirubin, albumin, and UA levels were found to be lower in newly diagnosed Crohn's patients who had not yet received medical treatment compared to the healthy controls, and this was associated with excessive consumption and destruction of serum bilirubin, albumin, and UA.

In a study by Zhu et al. (11) UA levels were found to be higher in inflammatory bowel disease compared to healthy controls, similar to present study, and it was observed that UA level increased with disease activity. UA is a parameter that undergoes renal elimination and is affected by renal functions. UA is a parameter that undergoes renal elimination and is affected by renal functions. Creatinine, on the other hand, is a biochemical parameter that changes depending on muscle mass and dietary protein, and it is a test that is affected by both kidney functions and nutritional status. For these reasons, in the same study, they evaluated the UA level, which is one of the parameters that will show the decrease in the endogenous antioxidant capacity of the patients, as the UA/creatinine ratio in order to minimize the interpretation errors. The lack of significant difference in UA level between the healthy controls and $\mathrm{CD}$ in the current study may be related to the medical treatment, the possible effect of medical treatment on the redox balance, or the fact that UA is a parameter affected by the nutritional status. In addition, oxidative stress management in the body is provided by enzymatic and non-enzymatic mechanisms, and the net effect of medical treatment on the enzymatic antioxidant system is unknown.

In the current study, a statistically significant difference was found between albumin and CRP levels and disease activity, but no significant difference was found between 
disease activity and total bilirubin, direct bilirubin, indirect bilirubin and UA levels. Kekilli et al. (12) found no difference in serum albumin levels between newly diagnosed active $\mathrm{CD}$ and $\mathrm{CD}$ patients in remission. The reason for this different result compared to the current study may be due to the low number of patients in remission in this study.

Albumin is a negative acute phase reactant and decreases as disease activity increases, while CRP level increases in line with the severity of inflammation. At the same time, albumin can be affected by the nutritional status of patients.

In the study of Su et al. (10) similar to present study, no significant difference was found between disease activity and UA level. They showed that as the severity of the disease increased, serum bilirubin, albumin and UA levels decreased, and CRP levels increased. The difference of this study from the current study is that newly diagnosed patients who have not yet received medical treatment were included in the study. The lack of a significant difference between disease activity and serum bilirubin and UA levels in the current study may be due to the small number of active patients. The fact that the patients were under medical treatment, and the different effects of the drugs used on the redox balance (10).

In the current study, in the comparison between remission and active Crohn's patients, CRP level was significantly higher and albumin level was significantly lower in patients with active disease. In contrast, no statistically significant difference was found between serum bilirubin and UA levels. The reason for these results is that all patients are receiving medical treatment and the number of active patients is relatively low. Serum bilirubin and albumin levels were found to be significantly lower in active Crohn's disease compared to healthy controls, but there was no statistically significant difference between UA values. If there is no difference between UA levels, it can be considered that all Crohn's patients should be under medical treatment. While there was a significant difference only in direct bilirubin levels between healthy controls and $\mathrm{CD}$ in remission, there was no significant difference in serum total bilirubin, indirect bilirubin, albumin, and UA levels.

In the light of this information, it can be concluded that redox balance is established in the body and serum antioxidant capacity reaches levels similar to the healthy population in patients who are in remission with medical treatment.

There is increased oxidative stress and decreased antioxidant capacity in active $\mathrm{CD}$, and serum antioxidant capacity reaches levels similar to the healthy controls in patients with remission (13-15).
In the study of Szczeklik et al. (16) serum bilirubin and albumin levels were found to be significantly lower in active $\mathrm{CD}$ compared to healthy controls and inactive $\mathrm{CD}$ patients, and present values were negatively correlated with disease severity. Lenicek et al. (17) reported that each $1 \mathrm{mmol} / \mathrm{L}$ decrease in serum bilirubin is associated with a $13 \%$ increase in the risk of developing CD. Schieffer et al. (18) suggested that the decrease in serum bilirubin is due to inflammation and that this phenomenon is observed not only in CD but also in various inflammatory diseases.

An Australian study (16) suggested that bilirubin levels are significantly lower in severe asthma, while inflammation affects antioxidant vitamins and bilirubin levels in asthmatics. This study shows the relationship between the changing serum concentration of bilirubin and albumin and oxidative imbalance in inflammation, similar to our study. In the study by Yang et al. (9) serum bilirubin, UA, albumin and creatinine levels were found to be lower in patients with myasthenia gravis, an autoimmune disease, when compared to the healthy controls. In the same study, when grouped according to the degree of disease progression, no significant difference was found in serum bilirubin levels, and serum UA, albumin and creatinine levels were negatively correlated with disease progression.

Peng et al. (19) found that serum total bilirubin, indirect bilirubin, UA and albumin levels were significantly lower in patients with neuromyelitis optica compared to healthy controls. Li et al. (20), found significantly lower serum bilirubin, UA and albumin levels in patients with newly was diagnosed pemphigus vulgaris that also known as a autoimmun severe bullous dermatosis compared to healthy controls. Albumin levels when compared to healthy controls.

Since CD is an inflammatory disease, it has been suggested that decreased antioxidant reserve is probably the pathogenic mechanism in the disease. Oxidative stress and antioxidant deficiency are thought to play a key role in the pathogenesis of CD-related gastrointestinal damage (15).

In the current study, did not find a significant difference in serum bilirubin, UA, and albumin levels between patients treated with azathioprine alone and those receiving other treatments. Neubauer et al. (20) investigated the possible relationship between non-enzymatic antioxidants and azathioprine treatment. In this study, no significant difference was found in serum UA levels between patients who received and did not receive azathioprine. Azathioprine may have opposite effects on systemic antioxidants. On the one hand, it causes an increase in systemic hydrogen peroxide, leading to cytotoxicity 
mediated by drug-induced oxidative stress in hepatocytes or T lymphocytes. On the other hand, azathioprine, which can undergo biotransformation through three different enzymatic systems and is metabolized by xanthine oxidase, produces thioric acid. Thioric acid is a compound equipped with a free sulfhydryl group, which has antioxidant capacity like UA. It has been shown in studies that sulfur derivatives of UA have neuroprotective effects and reduce ischemic brain damage (21).

Lemarachel et al. (22) measured protein oxidation levels in patients with rheumatoid arthritis who were treated with infliximab, and it was interpreted that redox balance was achieved due to the decrease in serum protein carbonyl groups after infliximab treatment. Monoclonal antibodies (anti-TNF- $\alpha$ ) inhibit the production of activated macrophage and other proinflammatory cytokines that increase $\mathrm{T}$ cell infiltration. A potent anti-inflammatory effect appears, including lysis of activated immune cells and induction of apoptosis by TNF- $\alpha$ neutralization (23).

Bednarek et al. (24) conducted a study on oxidative stress parameters in patients treated with corticosteroids for infiltrative Graves' ophthalmopathy. They found that oxidative stress parameters decreased during treatment and concluded that corticosteroids can temporarily reduce oxidative load. Corticosteroids are drugs that have anti-inflammatory and immunosuppressive effects by reducing lymphocyte proliferation and inhibiting inflammatory mediators secreted by macrophages. Thus, corticosteroids prevent the production of reactive free radicals. With the reduction of immune cells and mediators, the formation and release of free radicals is reduced, and oxidative stress is prevented.

There are some limitations in the current study. First, this is a retrospective study of patients with CD and has a small sample size. Secondly, due to the small number of patients with active $\mathrm{CD}$, it was prevented to reach clear conclusions in evaluating the relationship between bilirubin, albumin, UA and disease activation. Third, the relationship between some antioxidant enzymes (SOD, GSH-Px, catalase) and disease activity was not evaluated in the present study. Finally, a proportion of patients with CD have been treated with azathioprine, and the impact of this drug on outcomes cannot be excluded.

\section{CONCLUSION}

Albumin, bilirubin and UA, which are cost-effective tests that are routinely used in clinical practice, may be useful biomarkers in the detection of antioxidant status in Crohn's disease and may guide the early diagnosis of the disease. Larger-scale studies are needed for the use of these biomarkers for the evaluation of disease activity and treatment response.

\section{ETHICAL DECLARATIONS}

Ethics Committee Approval: The study was approved by Gazi University Faculty of Medicine, Clinical Research Ethics Committee (Date: 7/12/2020, Decision No: 834).

Informed Consent: Because the study was designed retrospectively, no written informed consent form was obtained from patients.

Referee Evaluation Process: Externally peer-reviewed.

Conflict of Interest Statement: The authors have no conflicts of interest to declare.

Financial Disclosure: The authors declared that this study has received no financial support.

Author Contributions: All of the authors declare that they have all participated in the design, execution, and analysis of the paper, and that they have approved the final version.

\section{REFERENCES}

1. Wilkins T, Jarvis K, Patel J. Diagnosis and management of Crohn's disease. Am Fam Physician 2011; 84: 1365-75.

2. Yang H, Li Y, Wu W, et al. The incidence of inflammatory bowel disease in Northern China: a prospective population-based study. PloS one 2014; 9: e101296.

3. Cosnes J, Gower-Rousseau C, Seksik P, et al. Epidemiology and natural history of inflammatory bowel diseases. Gastroenterology 2011; 140: 1785-94

4. Ananthakrishnan AN, Cheng SC, Cai T, et al. Serum inflammatory markers and risk of colorectal cancer in patients with inflammatory bowel diseases. Clin Gastroenterol Hepatol 2014; 12: 1342-8.

5. Jangi S, Otterbein L, Robson S. The molecular basis for the immunomodulatory activities of unconjugated bilirubin. Int J Biochem Cell Biol 2013; 45: 2843-51.

6. Dos Santos BH, de R Almeida CM, Skare TL. Systemic Lupus Erythematosus activity and serum bilirubins. Acta Reumatol Port 2013; 38: 242-6.

7. Peng Y, Zhang L, Pan G, et al. A potential clinical usefulness of measuring serum bilirubin levels in patients with polymyositis. Eur Rev Med Pharmacol Sci 2016; 20: 631-5.

8. Ames BN, Cathcart R, Schwiers E, et al. Uric acid provides an antioxidant defense in humans against oxidant-and radicalcaused aging and cancer: a hypothesis. Proc Natl Acad Sci USA 1981; 78: 6858-62.

9. Yang $\mathrm{D}, \mathrm{Su} \mathrm{Z}, \mathrm{Wu} \mathrm{S}$, et al. Low antioxidant status of serum bilirubin, uric acid, albumin and creatinine in patients with myasthenia gravis. Int J Neurosci 2016; 126: 1120-6.

10.Su Q, Li X, Mo W, et al. Low serum bilirubin, albumin, and uric acid levels in patients with Crohn's disease. Medicine (Baltimore) 2019; 98: e15664.

11.Zhu F, Feng D, Zhang T, et al.Altered uric acid metabolism in isolated colonic Crohn's disease but not ulcerative colitis. J Gastroenterol Hepatol 2019; 34: 154-61.

12. Kekilli M, Tanoğlu A, Karaahmet F et al. Midkine level may be used as a noninvasive biomarker in Crohn's disease. Turk J Med Sci 2020; 50: 324-9.

13. Boehm D, Krzystek-Korpacka M, Neubauer K, et al. Lipid peroxidation markers in Crohn's disease: the associations and diagnostic value. Clin Chem Lab Med 2012; 50: 1359-66.

14. Wendland BE, Aghdassi E, Tam C, et al. Lipid peroxidation and plasma antioxidant micronutrients in Crohn disease. Am J Clin Nutr 2001; 74: 259-64. 
15. Koutroubakis IE, Malliaraki N, Dimoulios PD, et al. Decreased total and corrected antioxidant capacity in patients with inflammatory bowel disease. Dig Dis Sci 2004; 49: 1433-7.

16. Szczeklik K, Krzyściak W, Cibor DA, et al. Evaluation of plasma concentrations of selected antioxidant parameters in patients with active Crohn's disease. Folia Medica Cracoviensia 2018; 58: 11930

17.Leníček M, Duuricová D, Hradsky O, et al. The Relationship Between Serum Bilirubin and Crohn's Disease. Inflamm Bowel Dis 2014; 20: 481-7.

18. Schieffer KM, Bruffy SM, Rauscher R, et al. Reduced total serum bilirubin levels are associated with ulcerative colitis. PloS one 2017; 12: e0179267.

19. Peng F, Yang Y, Liu J, et al. Low antioxidant status of serum uric acid, bilirubin and albumin in patients with neuromyelitis optica. Eur J Neurol 2012; 19: 277-83.

20. Li WC, Mo LJ, Shi X, et al. Antioxidant status of serum bilirubin, uric acid and albumin in pemphigus vulgaris. Clin Exp Dermatol 2018; 43: 158-63.

21.Neubauer K, Kempinski R, Matusiewicz M, et al. Nonenzymatic Serum Antioxidant Capacity in IBD and Its Association with the Severity of Bowel Inflammation and Corticosteroids Treatment. Medicina (Kaunas) 2019; 55: 88.

22.Lemarechal H, Allanore Y, Chenevier-Gobeaux C, et al. Serum protein oxidation in patients with rheumatoid arthritis and effects of infliximab therapy. Clinica Chimica Acta 2006; 372: 147-53.

23. Pereira C, Grácio D, Teixeira JP, et al. Oxidative stress and DNA damage: implications in inflammatory bowel disease. Inflamm Bowel Dis 2015; 21: 2403-17.

24. Bednarek J, Wysocki H, Sowiński J. Peripheral parameters of oxidative stress in patients with infiltrative Graves ophthalmopathy treated with corticosteroids. Immunol Lett 2004 93: 227-32. 